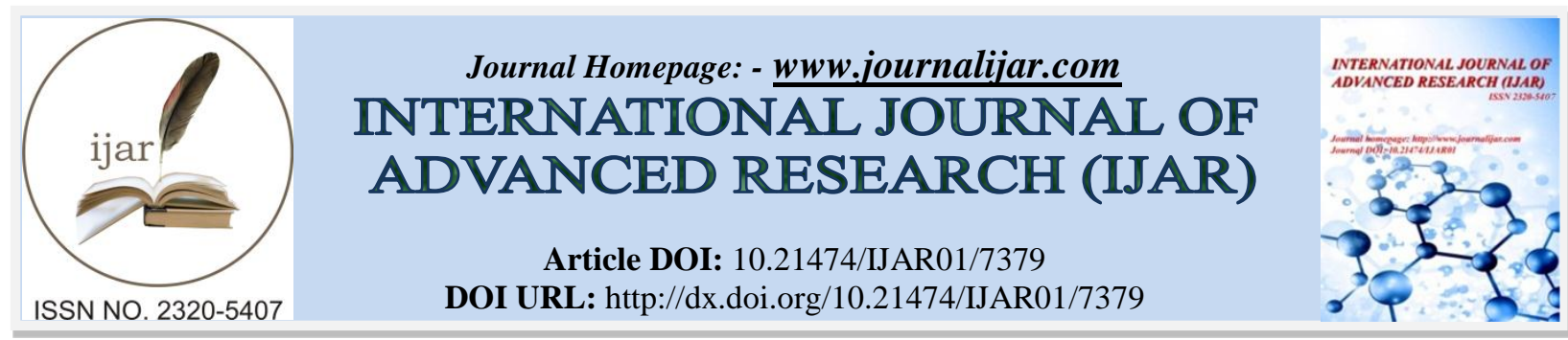

RESEARCH ARTICLE

\title{
CUTANEOUS PATHOMIMIA IN ADULTS: A SIGN OF PSYCHIATRIC ILLNESS.
}

Ahmed Bouhamidi' ${ }^{1}$ Youness Mamouni Alaoui ${ }^{2}$, Mohammed Boui ${ }^{1}$ and Naoufal Hjira ${ }^{1}$.

1. Department of Dermatology, Military Hospital of Instruction Mohammed V, Rabat, Morocco.

2. Department of Psychiatry, Military Hospital of Instruction Mohammed V, Rabat, Morocco.

\section{Manuscript Info}

Manuscript History

Received: 09 May 2018

Final Accepted: 11 June 2018

Published: July 2018

Keywords:-

Pathomimia, Psychiatric disorders, skin lesions.

\begin{abstract}
Psychocutaneous disorders are conditions characterized by psychiatric and skin manifestations, they are difficult to diagnose and a challenge to treat. Pathomimia is manifested by self-induced skin lesions, in order to attract more attention of the relatives and/or the medical staff. Depression and anxiety are the two most frequent psychiatric disorders among factitious disorders patients. Cooperation between dermatologists and psychiatrists, lead to significant improvement of those conditions. We report the case of a 67-year-old female with unknown psychiatric history and self-induced skin lesions of the lower limbs.
\end{abstract}

Copy Right, IJAR, 2018. All rights reserved.

\section{Introduction:}

Pathomimia also known as factitious disorders, are manifested by self-induced skin lesions in order to attract attention of family or medical staff. factitious disorders are classified as primary psychiatric disorders according to the DSM-V classification [1]. Depression and anxiety are the two most frequent psychiatric disorders among factitious disorders patients [2]. We report the case of a 67-year-old female with unknown psychiatric history and self-induced skin lesions of the lower limbs, and we insist therefore on the importance of the cooperation between dermatologists and psychiatrists, to diagnose these conditions, treat them and improve the quality of life of these patients.

\section{Clinical case:-}

A 67-year-old woman with unknown psychiatric problems, was hospitalized in our dermatology department for erosive skin lesions, covered with crusts, with discrete erythematous edges, accompanied by pruritus, located in the lower limbs (Figure 1). She described these lesions as being present for several months. She stated that she has already consulted many other dermatologists to resolve this problem without any clinical improvement despite the treatment received.

Cutaneous examination failed to find other skin lesions, except dystrophic nails related to a trichophyton rubrum infection, and actinic keratosis related to the patient's age. and routine blood tests were within the normal range.

A skin biopsy was performed, and the histopathological report excluded any of the known dermatosis.

A psychiatric consult was demanded, the patient admitted the self-mutilation by causing the skin lesions herself, she did not claim any delusions of parasitosis. The psychiatric examination confirmed a diagnosis of anxiety and depression provoked by some critical episodes in her life: a serious neurological disease of her child, and poor socio-

Corresponding Author:-Ahmed Bouhamidi.

Address:-Department of Dermatology, Military Hospital of Instruction Mohammed V, Rabat, 
economic condition. then, a systemic treatment with psychotropic drugs was initiate by psychiatrists (FLUOXETINE 20mg/day and BROMAZEPAM 6mg/day).

The erosive lesions were treated with topical medications such as topical steroids and fusidic acid cream, on occlusive dressings in order to minimize the possibility that the patient would manipulate the skin lesion.

Based on the history of the lesions, chronic evolution, and cutaneous damages displayed on attainable areas of the body, which did not mimic any of the known dermatosis, and associated with inconclusive skin biopsy, with significant clinical improvement after psychiatric therapy, we finally admitted the diagnosis of pathomimia, therefore the patient was transferred to the psychiatric department for followed up.

\section{Comments:-}

Pathomimia is a psycho-cutaneous disease, which include a large group of dermatological conditions characterized by skin lesions that patients inflict themselves, but deny their active participation [1].

Pathomimia are included in primary psychiatric disorders, and several studies show that they are related to serious psychiatric troubles such as personality disorders, schizophrenia, and dementia [2].

Although the overall incidence of factitious disorders is not known, and the number of patients requiring medical care for it is probably very important, partly because of a lack of knowledge of their clinical and psychological characteristics [3].

The diagnosis is often difficult, and can be a real challenge to dermatologists, because factitious disorders can be confused with other dermatosis, and patients are reluctant to admit the real origin of their lesions, and can lead to unnecessary analysis and treatments, thus delaying diagnosis and therapeutic measures in psychiatric units [4]. In this case, the clinical diagnosis was only obtained after a careful and multidisciplinary case study.

Indeed, the treatment cannot be separated from a psychiatric approach, which must support the dermatological treatments. Dermatological management of pathomimia is challenging and variable, and it depends on the anatomical site and how the patient produces the cutaneous manifestations. Sometimes it is also necessary to treat complications such as infections and scars [5] [6].

Affected areas are usually easy to reach with various objects such as knives and blades. Patients can also induce lesions simply with their hands or by self-injecting caustic or toxic substances into the skin [7] [8]. An occlusive dressing can be used, as it protects the patient from self-manipulation [9].

\section{Conclusion:-}

Skin lesions should be considered as a warning signal in critical cases with emotional and behavioral changes, and special attention should be given particularly to aged patients, while the dermatologist-psychiatrist cooperation is the main basis of the solving of these cases.

\section{Conflicts of interest:-}

The authors do not declare any conflict of interest for this work.

Authors' collaboration:-

All authors were actively involved in the development of this work. 




Figure 1:-Self-induced erosive skin lesions, covered with crusts, of the lower limbs. 


\section{References:-}

1. American Psychiatric Association. (2013). Diagnostic and statistical manual of mental disorders (5th ed.), Factitious Disorder DSM-5 300.19 (F68.10)

2. Mohandas P, Bewley A, Taylor R. Dermatitis artefacta and artefactual skin disease: the need for a psychodermatology multidisciplinary team to treat a diffcult condition. Br J Dermatol. 2013; 169:600-6.

3. Gattu S, Rashid RM, Khachemoune A. Self-induced skin lesions: a review of dermatitis artefacta. Cutis. 2009; 84:247-51.

4. Gregurek-Novak T, Novac-Bilic G, Vucić M. Dermatits artefacta: unusual appearance in an older woman. J Eur Acad Dermatol Venereol. 2005; 19:223-5.

5. Campanati A, Marconi B, Penna L, Paolinelli M, Ofdani A. Pronounced and early acne in Apert's syndrome: a case successfully treated with oral isotretinoin. J Dermatol. 2002; 12:496-8.

6. Koblenzer CS. Dermatitis artefacta. Clinical features and approaches to treatment. Am J Clin Dermatol. 2000; 1:47-55.

7. Ahmed A, Bewley A, Taylor R. Dermatitis artefacta in vulnerable adult with a dissociative state. Clin Exp Dermatol. 2013;38:921-3.

8. Campanati A, De Blasio S, Giuliano A, Ganzetti G, Giuliodori K, Pecora T, Consales V, et al. Response to Letter to the Editor: 'A precise knowledge of ozonated oils will help to define the favourable and peculiar properties of these functional dermatological matrices'. Burns. 2014. doi: 10.1016/j.burns.2013.12.013.

9. Campanati A, Savelli A, Sandroni L, Marconi B, Giuliano A, Giuliodori K, et al. Effect of allium cepaallantoin-pentaglycan gel on skin hypertrophic scars: clinical and video-capillaroscopic results of an open-label, controlled, nonrandomized clinical trial. Dermatol Surg. 2010; 36:1439-44. 